\title{
Vaccination against HPV-Associated Neoplasias
}

\section{Recommendations from the Current S3 Guideline of the HPV Management Forum of the Paul-Ehrlich Society - AWMF Guidelines, Registry No. 082-002 (short version), valid until Dec. 31st, 2018}

\author{
Impfprävention HPV-assoziierter Neoplasien \\ Empfehlungen aus der aktuellen S3-Leitlinie des HPV-Management Forums der Paul-Ehrlich-Gesellschaft \\ für Chemotherapie e. V. - AWMF Leitlinien-Register Nr. 082-002 (Kurzfassung), gültig bis 31.12.2018
}

Authors

Affiliations
G. Gross ${ }^{1}$, N. Becker ${ }^{2}$, N. H. Brockmeyer ${ }^{3}$, S. Esser ${ }^{4}$, U. Freitag ${ }^{5}$, M. Gebhardt ${ }^{6}$, L. Gissmann ${ }^{7}$, P. Hillemanns ${ }^{8}$, H. Grundhewer ${ }^{9}$, H. Ikenberg ${ }^{10}$, H. Jessen ${ }^{11}$, A. Kaufmann ${ }^{12}$, S. Klug ${ }^{13}$, J. P. Klußmann ${ }^{14}$, A. Nast ${ }^{15}$, D. Pathirana ${ }^{15}$, K. U. Petry ${ }^{16}$, H. Pfister ${ }^{17}$, U. Röllinghof ${ }^{18}$, P. Schneede ${ }^{19}$, A. Schneider ${ }^{20}$, E. Selka ${ }^{18}$, S. Singer ${ }^{21}$, S. Smola ${ }^{22}$, B. Sporbeck ${ }^{15}$, M. von Knebel Doeberitz ${ }^{23}$, P. Wutzler ${ }^{24}$

The affiliations are listed at the end of the article.

\section{50. Deutschsprachige Zusatzinformationen online abrufbar unter: www.thieme-connect.de/ ejournals/toc/gebfra}

\section{Bibliography}

DOI http://dx.doi.org/ 10.1055/s-0033-1360170

Geburtsh Frauenheilk 2014; 74: 233-241 @ Georg Thieme Verlag KG Stuttgart · New York ISSN 0016-5751

\section{Correspondence}

Prof. Dr. med. Gerd Gross

Klinik und Poliklinik für

Dermatologie und Venerologie

Universitätsklinikum Rostock

AöR

Strempelstraße 13

18057 Rostock

gerd.e.gross@t-online.de

\section{Abbreviations}

AIN Anal intraepithelial neoplasia

CIN Cervical intraepithelial neoplasia

HC2-Test Hybrid capture 2-test

HPV Human papilloma viruses

HR High-risk (high-risk HPV)

ICC Invasive cervical carcinoma

MSM Men who have sex with men

PCR Polymerase chain reaction

PIN Penile intraepithelial neoplasia

STIKO Standing committee on immunisation at the Robert Koch Institute

TVC Total vaccinated cohort

VAIN Vaginal intraepithelial neoplasia

VIN Vulvar intraepithelial neoplasia

VLP Virus-like Particles

\section{1}

\section{Introduction}

Neoplasias associated with anogenital human papilloma viruses (HPV) are characterised by high patient morbidity and mortality and by appreciable limitations in the patient's quality of life. Each year 530,000 women worldwide and 4800 women in Germany develop cervical cancer [1, 2]. Biomolecular and epidemiological studies carried out in the past 25 years have demonstrated causal link between persisting infections with HPV 16 and HPV 18 and at least 11 other so-called high-risk HPVs (HR-HPVs) and the development of cervical cancer and its precursor lesions (socalled dysplasias or, respectively, cervical intraepithelial neoplasias - CIN). HPV 16, HPV 18 and other HR-HPVs are also the causes of other cancers and their precursors, for example, vulvar, vaginal, penile and anal cancers as well as tonsil- lar and throat cancers and certain forms of skin cancer. So-called low-risk HPVs (LR-HPVs) such as HPV 6 and HPV 11 are responsible for over $90 \%$ of anogenital condylomata acuminata (anogenital warts). Condylomata acuminata are the most common viral sexually transmitted disease (STD) worldwide [3]. It is estimated that around $1 \%$ of European and German populations (aged 15-49 years) have these benign but often very unpleasant tumours. The development of a prophylactic quadrivalent vaccine (HPV 6, 11, 16, 18) and a bivalent vaccine (HPV 16, 18) has made it possible to prevent infections of the cervical epithelium and other squamous epithelia and the development of precancerous lesions. In the case of the quadrivalent vaccine (HPV 6, 11, 16, 18), the development of condylomata acuminata can also be prevented. The Standing Committee on Immunisation of the Robert Koch Institute (STIKO) has published a recommendation on HPV vaccination. Based on data from studies on the efficacy of HPV vaccines for the prevention of precancerous lesions of the cervix, vagina, and vulva, the STIKO recommends immunisation for girls aged between 12 and 17 years. The current guidelines do not contradict this recommendation but rather provide a more comprehensive supplement. The S3 guidelines focus on prophylactic vaccination against HPV-16 and HPV-18 or, respectively, HPV-6 and HPV-11 infections and thus on the prevention of cervical, vulvar, vaginal, anal and penile cancer and their precursors as well as on the primary prevention of condylomata acuminata and laryngeal papillomas. This S3 guideline thus clearly differs from other guidelines such as the S1 guideline "Condylomata acuminata and other HPV-associated clinical entities of the genitals, anus and urethra" (Guideline of the German 
STI Society in cooperation with the German Dermatological Society and the Paul-Ehrlich Society) and the S2 guideline of the German Society for Gynaecology and Obstetrics "Prevention, diagnostics and therapy for HPV infections and HPV-associated preinvasive lesions in gynaecology and obstetrics". After the debate on HPV immunisation has been concluded, the $\mathrm{S} 3$ guideline for the prevention of cervical cancer issued by the German Society for Gynaecology and Obstetrics will follow the recommendations in the current guideline, which will be updated. The development process and most important contents of the guideline are briefly described below. The long version of the guideline gives a more detailed account (www.awmf.org).

\section{Methods}

The present guidelines are an update of the first version of the S3 guideline on vaccination against neoplasias published in 2009 (short version) [4]. The core guideline group (HPV management forum) that initiated the update of this S3 guideline is a working group of the Paul-Ehrlich Society for Chemotherapy. It consists of a multidisciplinary group of experts whose members are appointed by various professional societies. In addition, an expanded, multidisciplinary panel of experts drawn from several relevant specialist medical societies also participated in the update. The composition of the guideline group is presented in - Table 1.
As part of the update, the HPV management forum formulated six key questions which were answered based on currently available evidence. All six key questions are clearly marked in the guideline and listed in the Methods report. A more recent systematic literature search using the databases Medline, Medline in Process, Embase and Cochrane Library was done with a closing date of 27.03.2012 to answer the six key questions. The closing date for the literature search of the initial guideline was 31.07.2007. A total of 665 publications were identified. After documentation and elimination of duplicates, the relevance of the articles for the guideline was evaluated on the basis of their abstracts. The full texts of 59 studies were acquired and evaluated using the inclusion criteria of this guideline to answer the key questions. After completion of this evaluation a total of 28 studies were included in the guideline and their methodological quality was assessed. These 28 studies served to answer the key questions and as a basis for part of the chapter "ADR/Safety". For each study included to answer the key questions, an evidence level was defined as follows as an indication of its quality:

A1 Meta-analyses that contained at least one randomised study at the $\mathrm{A} 2$ level where the results of the various studies were consistent.

A2 Randomised, double-blind clinical comparative studies of good quality (e.g., calculation of sample size, flow charts, ITT analysis, sufficient scope).

B Randomised, clinical studies of a less good quality or other comparative studies (not randomised: cohort or case-control studies).

C Non-comparative studies.

\section{Table 1 Composition of the group.}

\begin{tabular}{|c|c|}
\hline \multicolumn{2}{|l|}{ Members of the HPV management forum } \\
\hline Deutsche Dermatologische Gesellschaft e.V & Prof. Dr. med. Gerd Gross \\
\hline \multirow{4}{*}{ Deutsche STI-Gesellschaft e.V. } & Prof. Dr. rer. nat. Lutz Gissmann \\
\hline & Prof. Dr. med. Gerd Gross \\
\hline & PD Dr. med. Hans Ikenberg \\
\hline & Prof. Dr. med. K. Ulrich Petry \\
\hline \multirow[t]{2}{*}{ Paul-Ehrlich Gesellschaft für Chemotherapie e. V. } & Prof. Dr. med. Gerd Gross \\
\hline & PD Dr. rer. nat. Andreas Kaufmann \\
\hline Deutsche Krebsgesellschaft, AGO & Prof. Dr. med. Peter Hillemanns \\
\hline Deutsches Krebsforschungszentrum, Heidelberg & Prof. Dr. med. Magnus von Knebel Doeberitz \\
\hline \multirow[t]{2}{*}{ Deutsche Gesellschaft für Gynäkologie und Geburtshilfe e.V. } & Prof. Dr. med. K. Ulrich Petry \\
\hline & Prof. Dr. med. Achim Schneider \\
\hline \multirow[t]{2}{*}{ Gesellschaft für Virologie } & Prof. Dr. rer. nat. Dr. h. c. Herbert Pfister \\
\hline & Prof. Dr. med. Sigrun Smola \\
\hline Deutsche Gesellschaft für Urologie & Prof. Dr. med. Peter Schneede \\
\hline \multicolumn{2}{|l|}{ Members of the expanded expert group } \\
\hline \multirow[t]{2}{*}{ Deutsche Gesellschaft für Epidemiologie } & Prof. Dr. med. Nikolaus Becker \\
\hline & Prof. Dr. rer. nat. et med. habil. Stefanie Klug \\
\hline Deutsche Dermatologische Gesellschaft e. V. & Prof. Dr. med. N.H. Brockmeyer \\
\hline Deutsche STI-Gesellschaft e.V. & Prof. Dr. med. N. H. Brockmeyer \\
\hline Deutsche Gesellschaft für Koloproktologie & PD Dr. med. Daniel Dindo \\
\hline \multirow[t]{2}{*}{ Deutsche AIDS Gesellschaft e. V. } & Dr. med. Stefan Esser \\
\hline & Dr. med. Heiko Jessen \\
\hline Berufsverband der Frauenärzte & Dipl med. Ulrich Freitag \\
\hline Frauenselbsthilfe nach Krebs-BVe.V. & Marion Gebhardt \\
\hline Berufsverband für Kinder- und Jugendärzte & Dr. med. Herbert Grundhewer \\
\hline Deutsche Gesellschaft für Pathologie & Prof. Dr. med. Lars-Christian Horn \\
\hline Deutsche Gesellschaft für Medizinische Informatik, Biometrie und Epidemiologie e.V. & Prof. Dr. rer. nat. et med. habil. Stefanie Klug \\
\hline Deutsche Gesellschaft für Hals-Nasen-Ohrenheilkunde, Kopf und Hals-Chirurgie e. V. & Prof. Dr. med. Jens P. Klußmann \\
\hline \multirow[t]{2}{*}{ Deutsche Krebsgesellschaft, PRIO, PSO } & Prof. Dr. Karsten Münstedt \\
\hline & Prof. Dr. Susanne Singer \\
\hline \multirow[t]{2}{*}{ VulvaKarzinom-Selbsthilfegruppe e.V. } & Ulf Röllinghof \\
\hline & Enzia Selka \\
\hline Berufsverband Frauenärzte & Dr. med. Michael Wojcinski \\
\hline Deutsche Vereinigung zur Bekämpfung der Viruskrankheiten & Prof. Dr. med. Peter Wutzler \\
\hline
\end{tabular}


In addition, a standard of evidence was defined, which summarised the state of the evidence for each key question and which took account of the evidence levels of the individual studies:

1 Studies at evidence level A1 or studies with predominantly consistent results at evidence level A2;

2 Studies at evidence level A2 or studies with predominantly consistent results at evidence level B;

3 Studies at evidence level B or studies with predominantly consistent results at evidence level C;

4 Little or no systematic empirical evidence.

Based on the data on the key questions, evidence-based and nonevidence-based recommendations were discussed and agreed upon in formal consensus procedures in a nominal group process. Statements or recommendations for which no experimental scientific studies were available but which are generally followed and for which good agreement could be achieved within the consensus group are referred to as clinical consensus points (CCP, syn.: good clinical practice, GCP). The other aspects of the guideline were assessed based on the available literature without a systematic evaluation but taking account of the expert's many years of personal experience.

\section{Background}

\subsection{Virology and pathogenesis}

Following the initial determination of the DNA sequences of HPV in genital warts and in cervical cancer at the beginning of the 1980s, numerous biomolecular and epidemiological studies confirmed the relationship between HPV infection and the development of cervical dysplasias and cervical cancer. Moreover, a causative role of HPV is assumed for the majority of anal cancers and subgroups of vulvar, vaginal, penile and head and neck cancers [5]. Analogous conclusions with regard to the pathogenesis of these tumours are probably justified but require further substantiation. Today, at least 15 different so-called low-risk HPV types have been described as causes of genital warts or low-grade dysplasias. The role of 12 high-risk HPV types in the development of high-grade dysplasias or cervical cancer is considered to be certain; for 13 further types an involvement is assumed on the basis of limited epidemiological evidence or phylogenetic relationships with recognised or presumed high-risk HPV types [6]. HPV is transmitted by direct contact. Sexual contact is considered the main infection route for genital HPV types. Most HPV infections are recognised early and eliminated by the immune system, without progression to clinically relevant lesions [7]. In about $40 \%$ of cases the infection can persist for more than 6 months and eventually progress to a high-grade intraepithelial neoplasia [8]. Since high-risk HPV infection is one cause of cervical cancer [4], it can be assumed that prevention of the primary HPV infection will lead to a reduction in the incidence of cervical cancer and probably also that of other HPV-induced cancers.

\subsection{Vaccines/vaccine manufacturers and approval of vaccines}

Europe-wide, two HPV vaccines for the prevention of infections by certain HPV types have been approved by the European Medicines Agency. The approval covers the vaccination of girls and boys (quadrivalent vaccine) or, respectively, of girls (bivalent vaccine) from the age of 9 years upwards without an upper age limit. The STIKO has recommended use of this vaccination in Germany.
This recommendation currently applies to the vaccination of girls and young women aged from 12 to 17 years [9].

\subsection{Mechanism of action}

Vaccination with the prophylactic HPV-VLP vaccine generates serum antibodies with a titre that is more than 100 times higher than that occurring with a natural infection $[10,11]$. The antibodies are virus-neutralising, i.e., they prevent infection of epithelial cells through binding to the virus capsid. It is not yet known to what extent the cellular immune system with CD4 helper cells and CD8 cytotoxic T-cells plays a role in immunological memory and the prevention of persisting infection. In the currently available studies with $>25,000$ subjects high antibody titres were detected in $>99.9 \%$ of vaccinated subjects after the first immunisation.

\subsection{Dosage and timing of vaccination/duration of protection}

The clinical trials carried out and published to date on the efficacy of the vaccine involved women aged between 15 and 25 years. In addition, studies of healthy 9- to 15-year-old adolescents, boys and men as well as women up to the age of 55 have shown that the vaccination is generally well tolerated, highly immunogenic and, insofar as this can be measured, effective. At present the vaccination is recommended by the STIKO for girls/ young women aged between 12 and 17 years. If possible, vaccination should be completed (i.e., with three doses) prior to the first sexual intercourse. The vaccine has been approved for boys and girls aged 9 years and above without an upper age limit. An expansion of the recommendation to younger girls and boys could improve vaccination coverage and achieve a better herd immunity, probably however at the cost of its cost-efficiency. Factors which would support an early start to immunisation are: - Lower risk of prior exposure to HPV (first sexual intercourse),

- Higher immunogenicity in younger subjects and no evidence for poorer tolerance,

- Accessibility of the target group (compatible with vaccination scheme).

After the initial immunisation, it is recommended that the vaccination programme is completed after one (bivalent vaccine) or two (quadrivalent vaccine) months and again after six months. However, new data confirm that similarly high immune responses can be achieved in cases which deviated from the recommended scheme and when vaccinations are carried out at 0,6 and 12 months [12-14]. Those women who were not vaccinated in the period recommended by STIKO can still benefit from vaccination. Their physicians should assess the risks and benefits of vaccination and inform the patients accordingly taking their cue from the vaccine approval data [9]. In this context, data from investigations into the recurrence of dysplasias after conisation are of interest. In a retrospective comparison of patients from the phase III trials FUTURE I + II (quadrivalent vaccine) and PATRICIA (bivalent vaccine), a significant reduction of recurrent disease was found in the vaccinated group compared to the placebo group. After only 1.4 years' follow-up, an analysis of the FUTURE data reported a reduction in the incidence of recurrent disease of $65 \%$ [15]. Analysis of the PATRICIA data revealed a reduction of CIN2 or higher-grade lesions of $88.2 \%$ over the course of the study [16]. These data can be interpreted together with a higher reinfection rate for those patients who, apparently, develop effective immunity prior to conisation, who were rapidly re-infected thereafter and thus should be considered a special high-risk 
group. Data on the duration of vaccination protection cannot be expected from studies performed by manufacturers. Nevertheless, in a subgroup of women from a phase IIb trial followed up for more than 9.4 years, no breakthrough infection was seen whereas 4 cases of persisting infections occurred in the placebo group (difference not statistically significant) [17]. This correlates with a constant average antibody titre over time in vaccinated women that is much higher than that of a naturally acquired immune response [18]. Information on the duration of vaccine protection will only be available after many years from populationbased studies - if, in fact, an appreciable number of recurrent infections do occur after vaccination.

\subsection{Major contraindications/limitations for use}

No specific contraindications for the use of HPV vaccination are listed in the approval for the quadrivalent and bivalent vaccine $[19,20]$. Vaccination during pregnancy is not recommended because there is insufficient data to confirm that the vaccine is harmless. The HPV vaccine may be administered to breast-feeding women [9].

\section{$4 \quad$ Primary Prevention of CIN, Vulvar Dysplasias and Genital Warts}

An almost $100 \%$ vaccine protection against CIN caused by HPV 16 or HPV 18 has been demonstrated in women who were negative for HPV vaccine genotypes at the time of complete immunisation, i.e., after three doses of the vaccine (no evidence of disease found with PCR assay and patients were also seronegative) [21]. A partial vaccination protection of $44 \%$ has been observed after administration of at least one vaccine dose to women irrespective of their HPV status. Protection from vaccine-type associated VIN/ VAIN was also around $100 \%$ after prophylactic administration. Irrespective of the HPV status at the start of the trial, this protection decreased to $71 \%$ with regard to prevention of vaccine type-associated VIN/VAIN grade 2/3. Irrespective of HPV type, VIN/VAIN-1 lesions can be prevented in $18 \%$ and VIN/VAIN $2 / 3$ lesions in $26 \%$. Naturally induced antibodies against one HPV type do not react or react only very weakly against the capsids of other HPV types [22]. However, after vaccination antibody titres are appreciably higher than after natural infection. It has also been shown that induced antibodies can have a partially crossneutralising and cross-protective effect [23]. Cross-binding or cross-reactions have been demonstrated in the sera of women vaccinated with the quadrivalent vaccine. These sera contained antibodies that, although less effective, bound to the capsids of HPV types 31, 45, 52 and 58, which are closely related to HPV 16 and 18. In a pseudovirion neutralisation assay, it was shown in vitro that this cross-reaction also reduces infectivity [24]. Thus, vaccines provide greater protection than merely protection against the respective vaccine types. A partial cross-protection over and beyond the vaccine genotypes HPV 16 and 18 has been demonstrated for the closely related genotypes 31 and 45 in a phase II study with the bivalent vaccine [23]. Effectiveness was 50 and $90 \%$, respectively. These data were also supported by serological investigations into the cross-neutralising action of specifically induced antibodies. In a phase III study by Paavonen et al. [25], effectiveness with regard to prevention of six-month persistence of HPV types 45, 31, 33, 52 was 59.9\%, 36.1\%, 36.5\% and $31.6 \%$, respectively. New analyses of the end-of-study data from the PATRICIA trial showed a consistent effectiveness against per- sisting infections and CIN2 or higher grade lesions in sub-cohorts for HPV 31, 33, 45 and 51. Vaccine effectiveness against 12 HPV types not contained in the vaccine (HPV 31, 33, 35, 39, 45, 51, $52,56,58,59,66$, and 68 ) amounted to $46.8 \%$ for the accordingto-protocol (ATP) population, $56.2 \%$ in the TVC-naive population (all vaccinated subjects who were HPV-negative in month 7) and $34.2 \%$ for all subjects who had been vaccinated at least once. The effectiveness in the prevention of CIN 3 or higher grade lesions in the same groups amounted to $73.8 \%, 91.4 \%$ and $47.5 \%$, respectively.

\section{Primary Prevention of PIN and AIN}

AIN and anal cancer are a particular clinical problem in HPV-infected men with homosexual contacts; the corresponding AIN2/ 3 prevalence is more than $30 \%$ and the incidence of anal cancer is now about 100 -fold higher than in the heterosexual population. In more than $50 \%$ of all AIN2/3 lesions the DNA of the HPV 16 contained in the vaccine can be detected [26]. Similar numbers are seen for organ-transplanted subjects: an AIN was found in $20 \%(27 / 133)$ of investigated patients, and high-risk HPV type 16 was demonstrated in $47 \%$ of the patients [27]. The possible efficiency of the vaccine in HIV-infected MSM (men who have sex with men) is difficult to assess due to immunosuppression and multiple infections with high-risk HPV types. Multiple HPV infections have been identified in more than $60 \%$ of AIN2/3 lesions of HIV patients and HPV 16 is underrepresented relative to others $[26,28]$. The predominance of HPV 16 in anal cancer $(73 \%$ positive) supports the notion that, in spite of the presence of other HR-HPV infections, HPV 16 is mainly responsible for tumour progression. In recipients of organ transplants there are no appreciable differences in the extent of virus burden and the number of HPV types in comparison with healthy control subjects [29]. In a multinational study, Giuliano et al. [30] examined the quadrivalent vaccine in 4065 men aged 16-26 years. After an average follow-up period of 2.9 years, the per-protocol analysis revealed an effectiveness with regard to prevention of external genital lesions associated with HPV 6, 11, 16 and 18 (condylomas, PIN, perianal and perineal intraepithelial neoplasias) of $90.4 \%$ (95\% CI: $69.2-$ 98.1). Giuliano et al. [30] also investigated the effectiveness of the vaccine against PIN. An effectiveness of $100 \%$ was observed (95\% CI: - 3788.2-100.0) in the per-protocol analysis with regard to the prevention of PIN 2 or 3. In a sub-analysis of MSM in the same total population, Palewski et al. [31] calculated an effectiveness with regard to the prevention of HPV 6, 11, 16 und 18-associated AIN of $77.5 \%$ (95\% CI: 39.6-93.3) in the per-protocol analysis.

\section{$6 \quad$ HPV Tests Prior to Vaccination}

There is a clear recommendation for vaccination prior to first intercourse if possible. However, in practice the question of later vaccination is often posed and the question of HPV testing prior to vaccination also arises regularly. Although detection of HPV proteins or HPV-specific antibodies (serology) [32] or of the cytopathological effects caused by HPV are still not suitable or have not been sufficiently validated for routine diagnostics, the detection of HPV-RNA is considered sufficiently validated for this purpose [33]. One such procedure has already been recognised by the FDA [34]. Standardised methods for HPV-DNA detection are 
polymerase chain reaction (PCR) and hybridisation procedures with signal amplification. One prototype is the Hybrid-Capture2 (HC2) test. It is used to detect HPV types of high-risk (13 types) and low-risk groups (5 types). Recently, the separate detection of types 16, 18 and 45, which are all associated with the highest risk of progression, has become possible. In the meantime a second procedure based on signal amplification has been approved by the FDA [35]. In PCR assay, hybridisation with specific oligonucleotides, the primers, is done; the hybridisation product is subsequently amplified in reaction cycles. The sensitivity of PCR is very high; in theory a single molecule can be detected using this method. The very high analytical sensitivity of all PCR procedures for HPV, however, has not resulted in a higher sensitivity compared with HC2 for the detection of precancerous lesions and cancers in cancer screening. Because of the increased registration of clinically irrelevant HPV infections, early studies showed a marked reduction in the specificity of PCR in comparison to HC2 $[36,37]$. Detailed HPV typing in clinical routine currently seems to offer more disadvantages than advantages [38]. Results of trials into prophylactic HPV vaccines show that vaccination has no impact on already existing HPV infections [21]. Based on this assumption, it could be concluded that HPV testing should precede vaccination. This is currently not the case for the following reasons:

1. Persisting HPV infections are almost always single infections, so that in most cases protection against further types remains intact. Simultaneous infection with $4 \mathrm{HPV}$ types is present in only 1 of 10,000 cases. Complete inefficacy of the vaccination due to persisting infections is even less probable as the existing infection can offer cross-protection against other HPV types.
2. Comprehensive HPV screening in the age group 18 years and older would identify numerous transient infections that have no any clinical relevance; their identification would lead to considerable uncertainties for patients and their physicians. Not without reason do all experts only recommend the use of HPV tests in primary screening from the age of 30 year or more. Even then, this should be done using a test validated according to the guideline on account of the relatively high cutoff (and correspondingly high specificity).

3. If an HPV test result is available prior to vaccination it would appear to be reasonable in cases of HPV-HR positivity to perform a test for HPV 16 and 18. If this is negative, vaccination can be carried out. In cases of HPV 18 and, in particular, HPV 16 positivity, it is useful to repeat the test after 6-12 months and to vaccinate only those patients who are HPV 16 negative. 4. At present there are no tests for the serological detection of type-specific immune response to HPV vaccine types. The respective investigations in the vaccination trials were done using detection procedures that were developed by the manufacturers themselves which are not commercially available.

NB: In the presence of cytologically suspicious findings after vaccination, HPV tests are reasonable according to the recommendations of S2K Guideline [39]. Non-16 or 18 HPV types are detected in most cases. This provides a good argument to exclude an assumed vaccination failure.

In contrast, an HPV test to supplement cytology in cancer screening is reasonable from the age of 30 years onwards. In the presence of suspicious cytological or colposcopic findings or a suspicious case history, this is also valid for younger women. HPV testing is also indicated after therapy for CIN (see also the S2 guideline of the German Society for Gynaecology and Obstetrics (Deutsche Gesellschaft für Gynäkologie und Geburtshilfe)] [39].

\subsection{Recommendations for girls/young women}

\begin{tabular}{|c|c|c|}
\hline \multicolumn{3}{|l|}{ Recommendation 2} \\
\hline $\begin{array}{l}\text { Evidence-based recommendation (pre- } \\
\text { sentation of evidence, see long version) }\end{array}$ & All girls should be vaccinated as soon as possible after reaching 9 years of age. & Consensus \\
\hline \multicolumn{3}{|l|}{ Statement 1} \\
\hline $\begin{array}{l}\text { Evidence-based recommendation (pre- } \\
\text { sentation of evidence, see long version) }\end{array}$ & After starting sexual activities the expected benefits of vaccination will be reduced. & Majority agreement \\
\hline \multicolumn{3}{|l|}{ Recommendation 3} \\
\hline Clinical consensus point (CCP) & Decisions should be made on a case-by-case basis for these people. & Consensus \\
\hline
\end{tabular}

Recommendation 4

Clinical consensus point (CCP)
Treating a pre-existing CIN or ICC by vaccinating is not recommended since the efficacy of this approach has not been demonstrated.
Strong consensus 


\section{Statement 2}

Clinical consensus point (CCP)

* This statement is not supported by the following professional societies: Deutsche Gesellschaft für Epidemiologie, Deutsche Gesellschaft für Medizinische Informatik, Biometrie und Epidemiologie e.V.

\section{Recommendation 5}

Clinical consensus point (CCP)
HPV vaccination can be considered as an option prior to surgical treatment to reduce the risk of disease recurrence.
Majority agreement*

* This recommendation is not supported by the following professional societies: Deutsche Gesellschaft für Epidemiologie, Deutsche Gesellschaft für Medizinische Informatik, Biometrie und Epidemiologie e. V.

\subsection{Recommendations for men/male adolescents}

Recommendation 6
Evidenced-based recommendation $\quad$ All boys aged 9 years or more should be vaccinated as early as possible.
(for presentation of evidence,
see Section 5.3 )

$\begin{array}{ll}\text { Statement } 3 & \text { There is some evidence that disease recurrence after surgical treatment } \\ \text { Clinical consensus point (CCP) } & \text { Can be prevented in subjects who have received HPV vaccination. }\end{array}$

* This statement is not supported by the following professional societies: Deutsche Gesellschaft für Epidemiologie, Deutsche Gesellschaft für Medizinische Informatik, Biometrie und Epidemiologie e.V., Gesellschaft für Virologie

Recommendation 7

HPV vaccination can be considered as an option prior to surgical treatment

Consensus* to reduce the risk of disease recurrence.

* This recommendation is not supported by the following professional societies: Deutsche Gesellschaft für Epidemiologie, Deutsche Gesellschaft für Medizinische Informatik, Biometrie und Epidemiologie e.V., Gesellschaft für Virologie

\subsection{Recommendations for cancer screening}

\section{Recommendation 8 \\ Clinical consensus point (CCP)}

At present vaccinated women should continue to participate in cancer screening programmes because the currently available vaccines cannot prevent all oncogenic HPV infections.

\section{8}

\section{ADR/Safety}

It is the general opinion of the agencies approving and recommending HPV vaccination that the vaccine has an excellent safety profile and exhibits only few side effects. It has been demonstrated that the methodology and evaluation of the safety analyses are appropriate and in accordance with established procedure [17, $40]$. Very few participants $(0.2 \%)$ dropped out of the trials carried out to date due to side effects. These drop-outs occurred in both study arms and the numbers were comparable for the drug and placebo groups. No severe side effects that were definitively triggered by the vaccine were observed. Vaccination-typical acute local and systemic reactions did occur but were designated as mild to moderate and were of only transient duration ( $<3$ days). Local side effects at the injection site such as pain, reddening and swelling were common, i.e., occurred in over $10 \%$ of participants and were seen more often and were of a more severe nature in subjects receiving the vaccine compared to those receiving placebo. This was explained as a specific reaction against the antigen. Systemic side effects were fever, headache and nausea. Body temperatures of over $37.8^{\circ} \mathrm{C}$ were observed in about $10 \%$ of participants and temperatures of more than $38.9^{\circ} \mathrm{C}$ in about $1 \%$. The rates of side effects did not differ significantly between drug and placebo groups. Isolated cases $(<0.1 / 1000)$ of severe side effects possibly related to the vaccine were recorded and included bronchospasm, gastroenteritis, headache with high blood pressure and vaginal bleeding. Because of the low case numbers no conclusions about an increase after vaccination can be drawn. Only larger phase IV application studies will be able to confirm or exclude an association between such incidents and the vaccination. There were no deaths during the trials that could be connected to the vaccination. Between 2006 and 2009 Gee et al. [41] prospectively recorded the occurrence of severe diseases (Guillain-Barré syndrome [GBS], stroke, venous thromboembolism, appendicitis, seizure, syncope, allergic reactions and anaphylaxis) after vaccination with the quadrivalent vaccine. After more than 600,000 doses of vaccine their study found no statistically increased risk for any of the above-mentioned diseases. To date the maximum observation time after vaccination is 9.4 years. In this period, no increases in the frequency of newly occurring autoimmune diseases were observed in the study arms of various phase II and III trials. No statistically significant differ- 
ences were seen between study arms. The somewhat higher numerical occurrence of possible autoimmune diseases such as arthritis in the vaccine group remains within the range of variation for naturally occurring cases. Between June 2006 and October 2007 about 3500 potential side effects after the quadrivalent vaccine were reported to the internet-based US reporting system VAERS (Vaccine Adverse Event Reporting System), of which 347 were classified as serious. There were 3 deaths that occurred chronologically after vaccination. However, a causal relationship to the vaccination could not be detected, and other causes were found. On evaluation of the data on unwanted events after quadrivalent HPV vaccinations in the USA reported between 2006 and 2008 (VAERS data), a slightly higher notification rate was seen for venous thrombosis and syncope compared to other vaccinations [42]. The analyses carried out so far by the Center for Disease Control (CDC) and the US Food and Drug Administration FDA) have not revealed any increases in specific illnesses or complications, so that the HPV vaccine can still be classified as very safe and well tolerated. In a systematic review, Pomfret et al. [43] came to similar conclusions. They systematically evaluated the literature from 1966 to 2008. Both vaccines were considered to be safe, and most of the adverse effects were mild and transient. The reported serious effects were considered by the authors and the FDA to have occurred chronologically after vaccination but there was no causal relationship to the vaccination [43]. Lu et al. [44] performed a systematic meta-analysis of trials up until the middle of 2009 to assess the efficacy and safety of HPV vaccines. The cited studies showed a good tolerance, and severe adverse events were only rarely reported; there were isolated cases of complications during pregnancy. Since the introduction of HPV vaccination in Europe, two deaths have been reported that occurred subsequent to the quadrivalent vaccine vaccination in Germany and Austria. The case in Germany involved an 18-yearold woman who was found dead on the day after the second dose of the quadrivalent vaccine. The autopsy did not reveal any clear cause of death. In the second case of a 19-year-old woman in Austria, the first dose of the vaccine had been administered three weeks previously. Again, the autopsy did not reveal any remarkable findings, except for bronchitis. The German authorities (Paul-Ehrlich Institute), the vaccination committee of the Austrian Society for Paediatric and Adolescent Medicine (Gesellschaft für Kinder- und Jugendheilkunde) and the European approval agency (European Medicines Agency) did not find a causal relationship between the administration of HPV vaccines and the deaths of the two young women.

In discussions on vaccination, concerns about autoimmune diseases such as multiple sclerosis (MS) and Guillain-Barré syndrome have been voiced time and again. There have also been media reports about chronological connections between HPV vaccination and isolated cases of polyneuropathy and non-specific complications of strabismus.

The phase III trials of both vaccines showed no increased incidences in comparison with placebo. Chao et al. [45] investigated whether any of 16 different autoimmune diseases occurred in ca. 190,000 girls and women in the period of 180 days after quadrivalent HPV vaccination. There was no evidence of an increased occurrence of autoimmune diseases following vaccination. Isolated cases have been reported in the literature; these include a case of autoimmune hepatitis subsequent to vaccination [46], lipoatrophy at the injection site [47] and erythema multiforme [48, 49].
Souayah et al. [50] analysed the US data from the Vaccine Adverse Event Reporting System (VAERS) between 2006 and 2009 by investigating how often Guillain-Barré syndrome was reported subsequent to vaccination of the quadrivalent vaccine. They found a rate of 6.6 cases per 10 million in the 6 weeks after vaccination. This rate was slightly increased in comparison with other vaccinations and that of the age-matched average population [50]. There are several isolated case reports of demyelinising CNS diseases that occurred subsequent to HPV vaccination [5155]. To what extent they represent coincidental findings or whether there is, in fact, a causal relationship cannot be deduced from these individual observations. Certainly, more attention should be paid to these rare incidents. In the last report of the Paul-Ehrlich institute on pharmacovigilance in 2010, multiple sclerosis was reported in three patients after HPV vaccinations in the years 2007, 2008 and in one case with an unknown vaccination date. Causal relationships could not be assessed. A quantitative evaluation of the relative reporting frequency of multiple sclerosis in relation to HPV vaccination between 2006 and the end of 2010 was undertaken. No indications of a possible relationship to the vaccine were found [56].

In Great Britain seven cases of anaphylaxis after vaccination were reported between 2008 and 2009. Three of these cases occurred after bivalent HPV vaccination, which corresponds to a rate of 1.4 cases per one million doses of the vaccine. Almost all of these seven cases occurred in children who had previously already exhibited anaphylactic reactions, e.g., to foodstuffs [57]. In Australia, eight cases of anaphylaxis after vaccination with the quadrivalent vaccine were identified in 2007; this corresponds to a rate of 2.6 cases per 100,000 vaccine doses [58]. This rate and the reported British rate is slightly elevated in comparison with other vaccines. Overall, however, anaphylaxis after vaccination and especially after HPV vaccination is extremely rare.

HPV vaccination is mostly administered to young women who have an increased chance of becoming pregnant. No evidence that use of the vaccine in pregnant women could be unsafe has been found. The rate of pregnancies with a congenital anomaly amounted to 3-4\%; this was low and within the range of frequencies reported by surveillance registers. The numbers of spontaneous abortions, premature births and Caesarean sections were comparable in both trial arms. However, the existing studies were not designed to investigate vaccination in pregnant women. Thus, until evidence for the clinical safety of the vaccination becomes available in phase IV studies, use of the vaccine in pregnant women in Germany cannot be recommended. Vaccination during the breast-feeding period has not led to any severe vaccine-induced side effects in mother or baby.

Monitoring and recording potential side effects is important. However, in complete accordance with the European Medicines Agency, the Paul-Ehrlich Institute and the American Center for Disease Control, the HPV management forum recommends the unrestricted use of HPV vaccinations. Given the vaccination rates already achieved, statistically we must expect a number of unclear deaths as well as cases of autoimmune diseases subsequent to, but not caused by, the vaccination. 


\section{Conflict of Interest}

The Paul Ehrlich Society for Chemotherapy (Paul-Ehrlich-Gesellschaft für Chemotherapie e.V. [PEG]) provided the funding for the compilation of this Guideline. The Paul Ehrlich Society for Chemotherapy did not influence the contents of this Guideline in any way.

GG, LG, HI, AMK, HP, KUP, PS received honoraria from the companies GlaxoSmithKline and Sanofi Pasteur MSD. PH received honoraria from several companies currently working on HPV, cervical cancer, molecular diagnostics, cytology and vaccines. AS and SS declare that they have no financial relationships with any company whose products play an important role in this Guideline (or with any company which supplies rival products).

\section{Affiliations}

${ }^{1}$ Klinik und Poliklinik für Dermatologie und Venerologie, Universitätsmedizin Universität Rostock, Rostock

2 Deutsches Krebsforschungszentrum (DKFZ), Epidemiologie von Krebserkrankungen (C020), Heidelberg

${ }^{3}$ Klinik für Dermatologie und Allergologie der Ruhr-Universität, Bochum

${ }^{4}$ Klinik für Dermatologie und Venerologie, Universitätsklinikum Essen, Essen

${ }^{5}$ Frauenarztpraxis, Wismar

${ }^{6}$ Frauenselbsthilfe nach Krebs, Forchheim

${ }^{7}$ Deutsches Krebsforschungszentrum (DKFZ), FS Infektion und Krebs, Heidelberg

${ }^{8}$ Medizinische Hochschule Hannover (MHH), Frauenklinik, Abt. I für Frauenheilkunde und Geburtshilfe, Hannover

${ }^{9}$ Ausschuss Prävention des Berufsverbandes der Kinder- und Jugendärzte (BVKJ), Berlin

${ }^{10}$ MVZ für Zytologie und Molekularbiologie (CytoMol), Frankfurt/M.

11 Praxis Jessen + Kollegen, Berlin

12 Gynäkologische Tumorimmunologie, Gynäkologie mit Hochschulambulanz, Charité - Universitätsmedizin Berlin, Campus Benjamin Franklin, Berlin

${ }^{13}$ Universitätsklinikum Carl Gustav Carus an der Technischen Universität Dresden, Dresden

${ }^{14}$ Klinik und Poliklinik für Hals-Nasen-Ohrenheilkunde, Klinikum der Universität Gießen, Gießen

${ }^{15}$ Division of Evidence Based Medicine (dEBM), Klinik für Dermatologie, Allergologie und Venerologie, Charité - Universitätsmedizin Berlin, Campus Mitte, Berlin

${ }^{16}$ Klinikum Wolfsburg, Abteilung Gynäkologische Onkologie, Wolfsburg

17 Institut für Virologie der Universität zu Köln

18 VulvaKarzinom-SHG e.V. Wilhelmshaven

19 Klinikum Memmingen, Klinik für Urologie, Memmingen

${ }^{20}$ Klinik und Poliklinik für Gynäkologie, Charité - Universitätsmedizin Berlin, Campus Benjamin Franklin, Berlin

${ }^{21}$ Universitätsmedizin der Johannes Gutenberg-Universität Mainz, Institut für Medizinische Biometrie, Epidemiologie und Informatik, Abt. Epidemiologie und Versorgungsforschung, Mainz

22 Institut für Virologie, Institut für Infektionsmedizin, Universität des Saarlandes, Homburg/Saar

${ }^{23}$ Abteilung für Molekulare Pathologie, Pathologisches Institut des Universitätsklinikum Heidelberg, Heidelberg

24 Universitätsklinikum Jena (Friedrich-Schiller-Universität), Institut für Virologie und Antivirale Therapie, Beutenberg Campus, Jena

\section{References}

1 Arbyn M, Anttila A, Jordan J, Ronco G, Schenck U, Segnan $N$ et al. European guidelines for quality assurance in cervical cancer screening. 2nd revised ed. Bertrams; 2008; ISBN 978-92-79-07698-5

2 Jahn I, Eberle A, Niehues C, Birn A, Horch K. Gesundheitsberichterstattung des Bundes, Gebärmuttererkrankungen. Heft 37. Robert KochInstitut; 2007

3 Gross G, Tyring SK, eds. Sexually transmitted Infections and sexually transmitted Diseases. Springer; 2011; ISBN 978-3642146626

4 Pathirana D, Hillemanns P, Petry KU, Becker N, Brockmeyer NH, Erdmann $R$ et al. Short version of the German evidence-based Guidelines for prophylactic vaccination against HPV-associated neoplasia. Vaccine 2009; 27: 4551-4559

5 Cogliano V, Baan R, Straif K, Grosse Y, Secretan B, El Ghissassi F. Carcinogenicity of human papillomaviruses. Lancet Oncol 2005; 6: 204
6 Bouvard V, Baan R, Straif K, Grosse Y, Secretan B, El Ghissassi F et al. A review of human carcinogens - Part B: biological agents. Lancet Oncol 2009; 10: 321-322

7 Gravitt PE. The known unknowns of HPV natural history. J Clin Invest 2011; 121: 4593-4599

8 Schiffman M, Castle PE, Jeronimo J, Rodriguez AC, Wacholder S. Human papillomavirus and cervical cancer. Lancet 2007; 370: 890-907

9 Robert Koch-Institut. Mitteilung der Ständigen Impfkommission (STIKO) am Robert Koch-Institut: Impfung gegen humane Papillomaviren (HPV) für Mädchen von 12 bis 17 Jahren - Empfehlung und Begründung. Epidemiologisches Bulletin 2007: 12

10 Harper DM, Franco EL, Wheeler C, Ferris DG, Jenkins D, Schuind A et al. Efficacy of a bivalent L1 virus-like particle vaccine in prevention of infection with human papillomavirus types 16 and 18 in young women: a randomised controlled trial. Lancet 2004; 364: 1757-1765

11 Villa LL, Costa RL, Petta CA, Andrade RP, Ault KA, Giuliano AR et al. Prophylactic quadrivalent human papillomavirus (types $6,11,16$, and 18) L1 virus-like particle vaccine in young women: a randomised doubleblind placebo-controlled multicentre phase II efficacy trial. Lancet Oncol 2005; 6: 271-278

12 Esposito S, Birlutiu V, Jarcuska P, Perino A, Man SC, Vladareanu R et al. Immunogenicity and safety of human papillomavirus-16/18 AS04-adjuvanted vaccine administered according to an alternative dosing schedule compared with the standard dosing schedule in healthy women aged 15 to 25 years: results from a randomized study. Ped Inf Dis J 2011; 30: e49-e55

13 Neuzil KM, Canh do G, Thiem VD, Janmohamed A, Huong VM, Tang Yet al. Immunogenicity and reactogenicity of alternative schedules of HPV vaccine in Vietnam: a cluster randomized noninferiority trial. JAMA 2011; 305: 1424-1431

14 Zimmerman RK, Nowalk MP, Lin CJ, Fox DE, Ko FS, Wettick E et al. Randomized trial of an alternate human papillomavirus vaccine administration schedule in college-aged women. Journal of Women's Health (Larchmont) 2010; 19: 1441-1447

15 Joura EA, Garland SM, Paavonen J, Ferris DG, Perez G, Ault KA et al. Effect of the human papillomavirus (HPV) quadrivalent vaccine in a subgroup of women with cervical and vulvar disease: retrospective pooled analysis of trial data. Br Med J 2012; 344: e1401

16 Garland S; on behalf of the HPV PATRICIA Study Group. Does the HPV-16/ 18 AS04-adjuvanted vaccine benefit women with cervical disease? EUROGIN 2011; 2011: May 8-11, 2011; Lissabon

17 European Medicines Agency. Cervarix. 2012. Online: http://www.ema. europa.eu/ema/index.jsp?curl=pages/medicines/human/medicines/ 000721/human_med_000694.jsp\&mid=WC0b01ac058001d124\&murl=menus/medicines/medicines.jsp

18 Naud P, Roteli-Martins CM, De Carvalho N, Teixeira J, Borba P, Sanchez N et al. HPV-16/18 vaccine: sustained immunogenicity and efficacy up to 9.4 years. Berlin: 27th International papillomavirus conference and clinical workshop. 2011. Online: http://www.hpv2011.org/pics/1/4/ Abstract\%20Book\%202\%20APSC\%20WEBB\%20110922.pdf

19 GlaxoSmithKline. Fachinformation Cervarix. 2007. Online: http://www. fachinfo.de/data/fi/jsearch?praep

20 Sanofi Pasteur MSD. Fachinformation Gardasil. 2007. Online: http:// www.fachinfo.de/data/fi/jsearch?praep

21 FUTURE II Study Group. Quadrivalent vaccine against human papillomavirus to prevent high-grade cervical lesions. N Engl J Med 2007; 356: 1915-1927

22 Giroglou T, Sapp M, Lane C, Fligge C, Christensen ND, Streeck RE et al. Immunological analyses of human papillomavirus capsids. Vaccine 2001; 19: $1783-1793$

23 Harper DM, Franco EL, Wheeler CM, Moscicki AB, Romanowski B, RoteliMartins CM et al. Sustained efficacy up to 4.5 years of a bivalent L1 virus-like particle vaccine against human papillomavirus types 16 and 18: follow-up from a randomised control trial. Lancet 2006; 367: $1247-1255$

24 Smith JF, Brownlow M, Brown M, Kowalski R, Esser MT, Ruiz W et al. Antibodies from women immunized with Gardasil cross-neutralize HPV 45 pseudovirions. Human Vaccines 2007; 3: 109-115

25 Paavonen J, Jenkins D, Bosch FX, Naud P, Salmeron J, Wheeler CM et al. Efficacy of a prophylactic adjuvanted bivalent L1 virus-like-particle vaccine against infection with human papillomavirus types 16 and 18 in young women: an interim analysis of a phase III double-blind, randomised controlled trial. Lancet 2007; 369: 2161-2170 
26 De Vuyst H, Clifford GM, Nascimento MC, Madeleine MM, Franceschi S. Prevalence and type distribution of human papillomavirus in carcinoma and intraepithelial neoplasia of the vulva, vagina and anus: a metaanalysis. Int J Cancer 2009; 124: 1626-1636

27 Roka S, Rasoul-Rockenschaub S, Roka J, Kirnbauer R, Muhlbacher F, Salat A. Prevalence of anal HPV infection in solid-organ transplant patients prior to immunosuppression. Transplant International: Official Journal of the European Society for Organ Transplantation 2004; 17: 366-369

28 Wieland U, Stuecker M, Kreuter A. HPV vaccine against anal intraepithelial neoplasia. N Engl J Med 2012; 366: 378; author reply - 9

29 Busnach G, Piselli P, Arbustini E, Baccarani U, Burra P, Carrieri MP et al. Immunosuppression and cancer: a comparison of risks in recipients of organ transplants and in HIV-positive individuals. Transpl Proc 2006; 38: 3533-3535

30 Giuliano AR, Palefsky JM, Goldstone S, Moreira jr. ED, Penny ME, Aranda $C$ et al. Efficacy of quadrivalent HPV vaccine against HPV Infection and disease in males. N Engl J Med 2011; 364: 401-411

31 Palefsky JM, Giuliano AR, Goldstone S, Moreira jr. ED, Aranda C, Jessen H et al. HPV vaccine against anal HPV infection and anal intraepithelial neoplasia. N Engl J Med 2011; 365: 1576-1585

32 Wang SS, Schiffman M, Shields TS, Herrero R, Hildesheim A, Bratti MC et al. Seroprevalence of human papillomavirus-16, $-18,-31$, and -45 in a population-based cohort of 10000 women in Costa Rica. Br J Cancer 2003; 89: 1248-1254

33 Burger EA, Kornor H, Klemp M, Lauvrak V, Kristiansen IS. HPV mRNA tests for the detection of cervical intraepithelial neoplasia: a systematic review. Gynecol Oncol 2011; 120: 430-438

34 Castle PE, Dockter J, Giachetti C, Garcia FA, McCormick MK, Mitchell AL et al. A cross-sectional study of a prototype carcinogenic human papillomavirus E6/E7 messenger RNA assay for detection of cervical precancer and cancer. Clin Cancer Res 2007; 13: 2599-2605

35 Quigley NB, Potter NT, Chivukula M, Knight MZ, Welch JR, Olson MC. Rate of detection of high-risk HPV with two assays in women $>\mid=30$ years of age. J Clin Virol 2011; 52: 23-27

36 Bohmer G, van den Brule AJ, Brummer O, Meijer CL, Petry KU. No confirmed case of human papillomavirus DNA-negative cervical intraepithelial neoplasia grade 3 or invasive primary cancer of the uterine cervix among 511 patients. Am J Obstet Gynecol 2003; 189: 118-120

37 Snijders PJ, van den Brule AJ, Meijer CJ. The clinical relevance of human papillomavirus testing: relationship between analytical and clinical sensitivity. J Pathol 2003; 201: 1-6

38 Ikenberg $H$. Detection of HPV DNA and RNA. In: Pfister H, ed. Monographs in Virology: Prophylaxis and early Detection of HPV-related Neoplasia. Basel: Karger; 2011: 109-119

39 Deutsche Gesellschaft für Gynäkologie und Geburtshilfe (DGGG); Arbeitsgemeinschaft Infektiologie und Infektimmunologie in Gynäkologie und Geburtshilfe (AGII); Berufsverband der Frauenärzte (BVF); Deutsche Gesellschaft für Pathologie (DGP); Deutsche Gesellschaft für Urologie (DGU); Deutsche Krebsgesellschaft (DKG) et al. S2 k Leitlinie zur Prävention, Diagnostik und Therapie der HPV-Infektion und präinvasiver Läsionen des weiblichen Genitale. 2010. Online: http://www.dggg.de/fileadmin/public_docs/Leitlinien/1-4-4-hpv-2010.pdf

40 European Medicines Agency. Gardasil. 2012. Online: http://www.emea. europa.eu/ema/index.jsp?curl=pages/medicines/human/medicines/ 000703/human_med_000805.jsp

41 Gee J, Naleway A, Shui I, Baggs J, Yin R, Li R et al. Monitoring the safety of quadrivalent human papillomavirus vaccine: findings from the Vaccine Safety Datalink. Vaccine 2011; 29: 8279-8284
42 Slade BA, Leidel L, Vellozzi C, Woo EJ, Hua W, Sutherland A et al. Postlicensure safety surveillance for quadrivalent human papillomavirus recombinant vaccine. JAMA 2009; 302: 750-757

43 Pomfret TC, Gagnon JM, Gilchrist AT. Quadrivalent human papillomavirus (HPV) vaccine: a review of safety, efficacy, and pharmacoeconomics. J Clin Pharm Ther 2011; 36: 1-9

44 Lu B, Kumar A, Castellsague X, Giuliano AR. Efficacy and safety of prophylactic vaccines against cervical HPV infection and diseases among women: a systematic review \& meta-analysis. BMC Infect Dis 2011; 11: 13

45 Chao C, Klein NP, Velicer CM, Sy LS, SlezakJM, Takhar H et al. Surveillance of autoimmune conditions following routine use of quadrivalent human papillomavirus vaccine. J Int Med 2012; 271: 193-203

46 Della Corte C, Carlucci A, Francalanci P, Alisi A, Nobili V. Autoimmune hepatitis type 2 following anti-papillomavirus vaccination in a 11year-old girl. Vaccine 2011; 29: 4654-4656

47 Ojaimi S, Buttery JP, Korman TM. Quadrivalent human papillomavirus recombinant vaccine associated lipoatrophy. Vaccine 2009; 27: 48764878

48 Katoulis AC, Liakou A, Bozi E, Theodorakis M, Alevizou A, Zafeiraki A et al. Erythema multiforme following vaccination for human papillomavirus. Dermatology 2010; 220: 60-62

49 Perez-Carmona L, Aguayo-Leiva I, Gonzalez-Garcia C, Jaen-Olasolo P. The quadrivalent human papillomavirus vaccine: erythema multiforme and cutaneous side effects after administration. Dermatology 2010; 221: $197-200$

50 Souayah N, Michas-Martin PA, Nasar A, Krivitskaya N, Yacoub HA, Khan $H$ et al. Guillain-Barre syndrome after Gardasil vaccination: data from Vaccine Adverse Event Reporting System 2006-2009. Vaccine 2011; 29: 886-889

51 Alvarez-Soria MJ, Hernandez-Gonzalez A, Carrasco-Garcia de Leon S, del Real-Francia MA, Gallardo-Alcaniz MJ, Lopez-Gomez JL. Trastornos neurologicos desmielinizantes y vacunacion del papilomavirus humano [Demyelinating disease and vaccination of the human papillomavirus]. Revista de Neurologia 2011; 52: 472-476

52 Bomprezzi R, Wildemann B. Acute disseminated encephalomyelitis following vaccination against human papilloma virus. Neurology 2010; 74: 864-865

53 Chang J, Campagnolo D, Vollmer TL, Bomprezzi R. Demyelinating disease and polyvalent human papilloma virus vaccination. J Neurol Neurosurg Psychiatry 2011; 82: 1296-1298

54 DiMario FJ, Hajjar M, Ciesielski T. A 16-year-old girl with bilateral visual loss and left hemiparesis following an immunization against human papilloma virus. J Child Neurol 2010; 25: 321-327

55 Wildemann B, Jarius S, Hartmann M, Regula JU, Hametner C. Acute disseminated encephalomyelitis following vaccination against human papilloma virus. Neurology 2009; 72: 2132-2133

56 Mentzer D, Keller-Stanislawski B. Daten zur Pharmakovigilanz von Impfstoffen aus dem Jahr 2010. Bulletin zur Arzneimittelsicherheit 2012: $21-29$

57 Erlewyn-Lajeunesse M, Hunt LP, Heath PT, Finn A. Anaphylaxis as an adverse event following immunisation in the UK and Ireland. Arch Dis Child 2012; 97: 487-490

58 Brotherton JML, Gold MS, Kemp AS, McIntyre PB, Burgess MA, CampbellLloyd $S$. Anaphylaxis following quadrivalent human papillomavirus vaccination. Can Med Ass J 2008; 179: 525-533 